quadrantic biopsies. DNA content abnormalities (aneuploidy/tetraploidy); loss of heterozygosity (LOH) at 9p and 17p loci; RUNX3, HPP1 and p16 methylation; immunohistochemistry (IHC) for p53 and Cyclin A were tested on targeted biopsies. Each biomarker was correlated with the dysplasia and AFI status.

Results 111 patients with 210 biopsy areas were included in the analysis (AFI+, $\mathrm{n}=120$; AFI-, $\mathrm{n}=90$ ). Univariate per-biopsy analysis showed that all biomarkers correlated with dysplasia $(p<0.05)$, with exception of $9 \mathrm{p} \mathrm{LOH}$. Multivariate analysis showed that aneuploidy, p53 IHC and Cyclin A (3 biomarker panel) were independently associated with dysplasia with an $\mathrm{AUC}=0.93$ (95\% CI 0.88 to 0.98$)$ for any dysplasia and AUC=0.95 (95\% CI 0.89 to 1) for HGD/early cancer (EC). AFI positivity significantly correlated with aneuploidy, p16 methylation, cyclin A and p53 staining $(p<0.05)$. After excluding dysplastic areas, aneuploidy $(p=0.03)$ and $p 53(p=0.04)$ staining retained statistical correlation with AFI positivity. Analysis of the 3 biomarker panel in patients with dysplasia showed significant biomarker enrichment in AFI+ compared to AFI- areas $(p=0.001)$. Finally, 3 biomarker panel was used to predict prevalent dysplasia. Using a cut-off of $\geq 2$ biomarkers, the panel when applied to AFI+ areas alone, showed sensitivity and specificity of $88 \%$ and $90 \%$ respectively for diagnosis of $\mathrm{HGD} / \mathrm{EC}$, and $64 \%$ and $96 \%$ respectively for diagnosis of any dysplasia, compared to overall histology.

Conclusion AFI increases detection rate for molecular biomarkers. A panel of 3 molecular biomarkers on a small number of AFI targeted biopsies can efficiently predict the dysplasia status and potentially inform therapeutic management of patients with BE.

Competing interests None declared.

\section{PTU-193 GASTRO-0ESOPHAGEAL REFLUX (GORD) AND COELIAC DISEASE: A BIDIRECTIONAL STUDY}

doi:10.1136/gutjnl-2012-302514c.193

${ }^{1} \mathrm{~K}$ Evans, ${ }^{*}{ }^{1} \mathrm{~S}$ M Barrett, ${ }^{1,2} \mathrm{~A}$ Smythe, ${ }^{1} \mathrm{D}$ S Sanders. ${ }^{1}$ Sheffield Teaching Hospitals NHS Trust, Sheffield, UK; ${ }^{2}$ University of Sheffield, Sheffield, UK

Introduction There are limited data assessing the relationship between coeliac disease and GORD. We aimed to establish the prevalence and nature of GORD symptoms in patients with coeliac disease, and the prevalence of undetected coeliac disease in those presenting to endoscopy with GORD symptoms.

Methods Group A were histologically proven patients with coeliac disease $(n=225)$ who were asked to complete a validated reflux questionnaire and then compared to age/sex-matched controls $(\mathrm{n}=348)$. Group B were consecutive GORD patients undergoing endoscopy who had duodenal biopsies and coeliac serology taken. $(n=851)$ Furthermore, patients with newly diagnosed coeliac disease underwent manometry and $\mathrm{pH}$ studies prior to commencing gluten free diet $(\mathrm{n}=33)$.

Results In Group A the prevalence of GORD was greater in coeliac disease $(66 \%)$ than in healthy controls $(50 \%) \mathrm{p}=0.0001$. Coeliac patients also report reflux of greater severity: coeliac disease $\mathrm{OR}=6.8,95 \% \mathrm{CI}=3.6$ to $12.7, \mathrm{p} \leq 0.001$. In Group B at endoscopy the prevalence of undetected coeliac disease was $1.66 \%$ (14/851). In Group C 31/33 were able to tolerate manometry and complete testing (2 had partial investigation before the catheter was removed) At manometry 30/33 (91\%) had a normal lower oesophageal sphincter (LOS). 2/33 had a hypotensive LOS. 21/33 (64\%) had normal oesophageal motility. However, 10/33 had a hypocontractile oesophagus, 1 was hypertensive and 1 showed functional oesophagogastric junction obstruction. During manometry 6/31 (19\%) demonstrated significant reflux, 6/31 (19\%) had some reflux and the final $19 / 31(61 \%)$ had no reflux episodes. In these coeliac patients neither the presence of symptoms nor abnormal oesophageal study findings was related to histological grade of coeliac disease, (villous atrophy) or serology findings.

Conclusion Up to two thirds of patients with coeliac disease report reflux symptoms and one third have demonstrable abnormalities of oesophageal motility and reflux. However, in an unselected population at endoscopy, reflux symptoms are not predictive of coeliac disease.

Competing interests None declared.

\section{PTU-194 DOES METABOLIC SYNDROME IMPACT TUMOUR PATHOLOGY IN OESOPHAGEAL ADENOCARCINOMA?}

doi:10.1136/gutjnl-2012-302514c.194

${ }^{1} \mathrm{~L}$ A Healy, * ${ }^{2} \mathrm{~J}$ Howard, ${ }^{2} \mathrm{~J}$ V Reynolds. ' Clinical Nutrition, St James Hospital, Dublin 8, Ireland; ' Surgery, St James Hospital, Dublin 8, Ireland

Introduction Obesity is an established risk factor for both the increased incidence of oesophageal adenocarcinoma cancer (OAC), and adverse outcomes by increasing risk of recurrence and reducing survival in obese patients post oesophagectomy. The exact mechanism of this relationship is unclear but the pattern of fat distribution pattern is likely important. Abdominal obesity more closely reflects an increased visceral fat area and is associated with alterations in metabolic risk profile. The clustering of central obesity, hypertension, and raised plasma glucose, triglycerides and HDL cholesterol is also known as the metabolic syndrome (MetS). The processes underling the metabolic syndrome especially insulin resistance and increased leptin, can provide a favourable growth environment for malignant cells and may have a role in cancer progression.

Methods The aim of this prospective observational study of OAC patients was to examine the incidence of MetS and its relationship to tumour pathology in an Irish population. Patients underwent a metabolic and nutritional assessment prior to initiation of treatment. Visceral fat area was measured using CT scans. MetS was defined according to the International Diabetes Federation definition. ${ }^{1}$

Results 83 OAC patients ( 71 male: 12 female) were recruited with a median age of 64.6 years \pm 1.0 (range $48-86$ ). All patients underwent an oesophagectomy, with $42 \%(n=35)$ receiving neoadjuvant chemoradiotherapy. $58 \%$ of patients were either overweight or obese with a further $60 \%$ centrally obese. Males had significantly greater visceral fat area $(p=0.031)$ despite no difference in total abdominal fat compared to females $(p=0.757)$. The incidence of obesity may be underestimated as $41 \%$ of patients reported unintentional weight loss with $18 \%$ losing $>5 \%$ of their usual body weight. MetS was diagnosed in $39 \%$ patients, which exceeds the population norms reported at $21 \%{ }^{2}$ The presence of MetS was not associated with tumour length, depth of invasion, lymph node positive disease, clinical or overall pathological stage in males or females. Individual features of MetS were also not significantly related to the pathological staging of oesophageal cancer.

Conclusion We report an increased prevalence of MetS and central obesity in a cohort of Irish patients with OAC. Dysphagia and weight loss are common in the presentation of oesophageal cancer and may mask the effect of obesity and metabolic syndrome on the clinical pathological features of OAC in this cohort. Further research is needed to fully understand the underlying biological mechanisms linking obesity to oesophageal cancer. 